Char act eri st i cs of I ong- pul se negat i ve- i on source i $n$ the neut $r$ al beami nj ect or of Large Hel i cal Devi ce

\begin{tabular}{|l|l|}
\hline $\begin{array}{l}\text { jour nal or } \\
\text { publ i cat i on } \mathrm{titl} \text { e }\end{array}$ & Revi ew of Sci ent i f i c I nst r ument s \\
\hline vol une & Vol . 77 \\
\hline number & I ssue3 \\
\hline page r ange & pp. 03A523-1 03A523-4 \\
\hline year & 2006- 03- 01 \\
\hline URL & ht t p: //hdl . handl e. net /10655/3867 \\
\hline
\end{tabular}




\title{
Characteristics of long-pulse negative-ion source in the neutral beam injector of Large Helical Device
}

\author{
Y. Takeiri, K. Ikeda, Y. Oka, K. Tsumori, M. Osakabe, K. Nagaoka, O. Kaneko, E. Asano, \\ T. Kondo, M. Sato, M. Shibuya, and S. Komada \\ National Institute for Fusion Science, Toki 509-5292, Japan
}

(Presented on 15 September 2005; published online 21 March 2006)

\begin{abstract}
The injection duration has been extended beyond $100 \mathrm{~s}$ with a high-power hydrogen negative-ion source in a negative-ion-based neutral beam injector of the Large Helical Device superconducting fusion machine. The ion source is a cesium-seeded source with a thermally insulated plasma grid $(\mathrm{PG})$, and optimized for a short-pulse operation of 2-3 s. The negative-ion production efficiency is strongly dependent on the PG temperature, and in the long-pulse operation it exceeds an appropriate temperature range of $200-300{ }^{\circ} \mathrm{C}$, at which the optimum cesium coverage is formed on the PG surface. By making the PG temperature rise slower with a reduced arc power, the injection duration was extended to $110 \mathrm{~s}$ with an injection power of $110 \mathrm{~kW}$. To extend the injection duration further with a higher injection power, stainless-steel cooling tubes have been mechanically attached to the PG for suppression of the PG temperature rise in the long-pulse operation. As a result, a long-pulse injection with an injection power of $200 \mathrm{~kW}$ was extended to $128 \mathrm{~s}$ until it was manually stopped due to the plasma collapse. However, the beam duration could be limited to around 3 min because the PG temperature rise was not saturated due to a low thermal conductivity with the thickness of the stainless-steel tube determined so that the short-pulse operation is also possible. On the other hand, the longitudinal beam distribution in a grid area of $25 \times 125 \mathrm{~cm}^{2}$ is observed to be more uniform than that with the uncooled PG. The temperature distribution of the individual grid parts becomes more uniform with the cooled PG, which should contribute to the improvement of the beam uniformity. (C) 2006 American Institute of Physics. [DOI: 10.1063/1.2166675]
\end{abstract}

\section{INTRODUCTION}

High-power hydrogen negative-ion sources are operated routinely in negative-ion based neutral beam injectors (NBI) of the Large Helical Device (LHD) ${ }^{1}$ which is the world's largest superconducting fusion machine. ${ }^{2}$ The negative-ion source is a cesium-seeded source, ${ }^{3}$ and the energy and current of the negative-ion beam produced reach the design values of $180 \mathrm{keV}$ and $30 \mathrm{~A}$, respectively, in a short-pulse operation of 2-3 s. ${ }^{4}$ However, extension of the pulse duration has been limited to several tens of seconds ${ }^{5}$ because the temperature of the plasma grid (PG) rises beyond an appropriate temperature of $200-300{ }^{\circ} \mathrm{C}$ in the long-pulse shots, at which optimum cesium coverage is formed on the PG surface for efficient negative-ion production. The PG is thermally insulated to maintain the appropriate temperature for the shortpulse operation of 2-3 s every $3 \mathrm{~min}$. By making the PG temperature rise slower with a reduction of the arc power, the injection duration has been extended to $110 \mathrm{~s}$ although the injection power was much reduced. In order to suppress the PG temperature rise in the long-pulse operation, stainlesssteel cooling tubes have been mechanically attached to the PG instead of the structure of thermally insulated PG. As a result, the injection duration was extended to $128 \mathrm{~s}$ with a higher injection power.

In this article, the operational characteristics in the longpulse injection with the negative-ion source in the LHD negative-NBI are presented. In the next section, the structure of the negative-ion source is briefly outlined, including the cooled PG for the long-pulse operation. Then, the ion source performance during the long-pulse injection is described in relation to the PG temperature, which has a great influence on the negative-ion production efficiency in the cesiumseeded source. Finally, the beam uniformity is discussed in the long-pulse ion source with the cooled PG.

\section{STRUCTURE OF ION SOURCE}

The cesium-seeded negative-ion source used in the LHD-NBI system employs a large filament-arc plasma source with an external magnetic filter. ${ }^{3}$ The arc chamber is rectangular and has large dimensions of $35 \mathrm{~cm}$ (width) $\times 145 \mathrm{~cm}$ (length) $\times 21 \mathrm{~cm}$ (depth), which is surrounded by a strong cusp magnetic field. The external magnetic filter is transverse to the width direction with a magnetic-field strength of $52 \mathrm{G}$ at the center. Thick tungsten filaments of 1.8 $\mathrm{mm}$ in diameter are used for the arc discharge. Three cesium ovens are attached to a back plate of the arc chamber, and cesium vapor is injected remotely through an air-actuated valve. The negative-ion accelerator is a single-stage acceleration system, consisting of three grids of PG, extraction grid (EG), and grounded grid (GG). The grid area is $25 \mathrm{~cm}$ (width) $\times 125 \mathrm{~cm}$ (length) and is segmented into five parts longitudinally, which are jointed so that each part is aimed at a focal point $13 \mathrm{~m}$ downstream. The PG is made of molybdenum and is thermally insulated to keep the PG temperature 


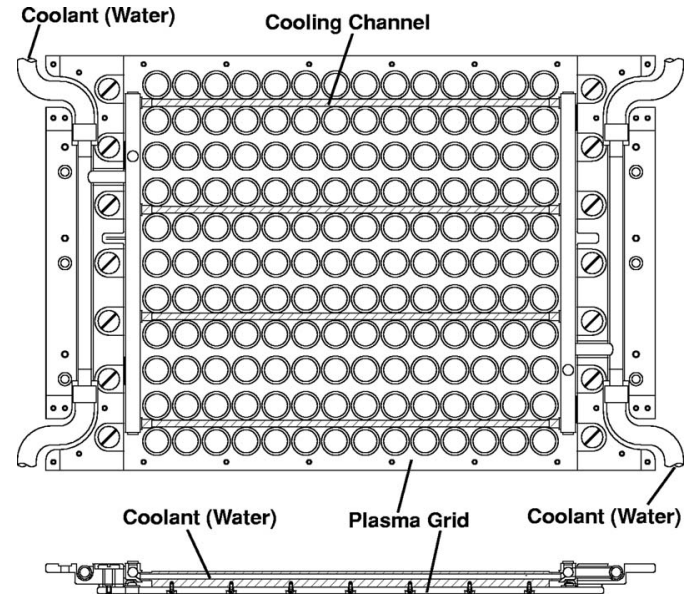

FIG. 1. Drawing of one part of the segmented cooled plasma grid. The stainless-steel cooling channels are mechanically attached to the grid surface.

above $200{ }^{\circ} \mathrm{C}$ for a short-pulse operation of $2-3$ s every 3 min. The EG contains permanent magnet bars in the width direction for electron suppression. The detailed structure of the ion source is described in Ref. 3.

The structure of the PG has been modified so that the PG temperature rise would be suppressed in long-pulse operation over 100 s. Figure 1 shows the structure of the modified PG for the long-pulse operation. Stainless-steel cooling tubes are mechanically attached to the PG, and the thickness between the cooling channel and the contact surface is determined so that the heat diffusivity to the PG is lower than that of the molybdenum PG itself. By this structure, the distribution of the segmented PG temperature should be uniform. The thickness of the cooling tube is relatively thick to have a thermal insulation to such an extent that the short-pulse injection would also be possible by extending the prearc discharge duration before the beam extraction. In actual short-pulse operation with this cooled PG, the prearc discharge duration has been extended to around $15 \mathrm{~s}$ from $5-7 \mathrm{~s}$ with the uncooled PG.

The PG temperature rise as a function of the arc discharge duration for the uncooled and cooled PG are shown in Fig. 2. With the cooled PG, the PG temperature is increased

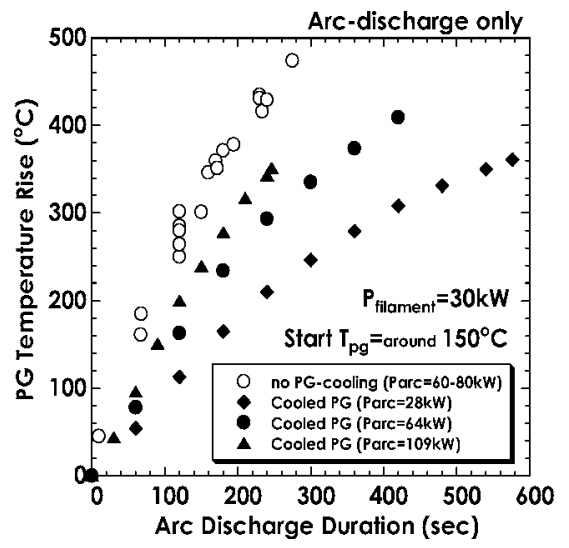

FIG. 2. PG temperature rises of the uncooled and cooled PG as functions of the arc discharge duration for various arc powers. The PG temperature is around $150{ }^{\circ} \mathrm{C}$ at the start of the arc discharge.
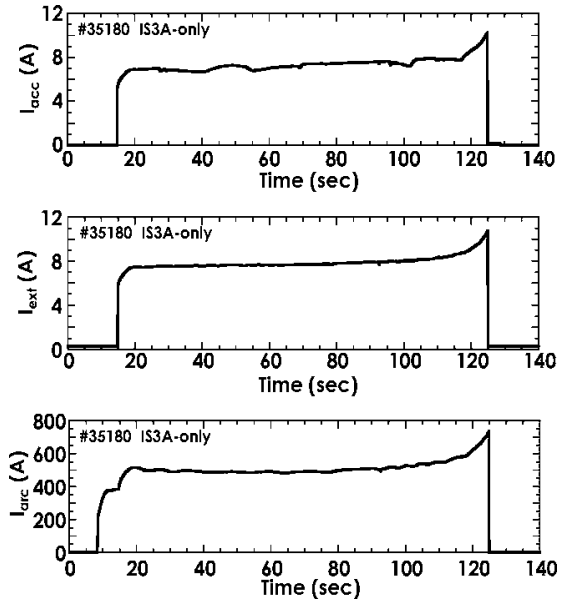

FIG. 3. Time variations of the acceleration and extraction drain currents and the arc current in a long-pulse shot for $110 \mathrm{~s}$. The uncooled plasma grid is used. The beam energy is $80 \mathrm{keV}$ and the injection power is about $110 \mathrm{~kW}$.

more slowly at the same arc power. While it takes about $80 \mathrm{~s}$ for the PG temperature rise to exceed $200{ }^{\circ} \mathrm{C}$ with the uncooled PG, the arc duration is extended to about $150 \mathrm{~s}$ to exceed $200{ }^{\circ} \mathrm{C}$ with the cooled PG for nearly the same arc power of around $70 \mathrm{~kW}$. However, since the cooled PG is not actively cooled, the temperature rise is not saturated, and the injection duration would not be extended beyond several minutes.

\section{CHARACTERISTICS OF LONG-PULSE OPERATION}

The power supply system for the negative-ion source was originally designed for a maximum beam-pulse length of $10 \mathrm{~s}$, while the main beamline components were designed for continuous operation except for the injection port covered annularly with inertially cooled molybdenum plates. By modification of the power supply system, the beam-pulse length can be extended to over $10 \mathrm{~min}$ at a reduced power. However, the ion source is optimized for the pulsed operation for several seconds with regard to control of the PG temperature for the efficient negative-ion production in the cesium-seeded mode. This limits the present long-pulse operation of the negative-ion source because the PG is thermally insulated to maintain an appropriate temperature of 200-300 ${ }^{\circ} \mathrm{C}$ in the short-pulse operation. To extend the beampulse duration with the uncooled PG, the rise rate of the PG temperature was lowered by reducing the arc power to 32 $\mathrm{kW}$, lower than a quarter of that in the high-power shortpulse shots. As a result, the injection duration was extended to $110 \mathrm{~s}$ with the original uncooled PG. Figure 3 shows the time variations of the acceleration and extraction drain currents and the arc current in the long-pulse shot. The beam energy is $80 \mathrm{keV}$ and the injection power is about $110 \mathrm{~kW}$. The PG temperature is monotonously increased and exceeds $350{ }^{\circ} \mathrm{C}$ at the end of the shot. As shown in Fig. 3, the arc current is gradually increased after $t=100 \mathrm{~s}$, followed by a gradual increase in the extraction current. The gradual increase in the arc current is probably due to the out gas including the cesium evaporation deposited on colder surfaces in short-pulse operation. Deterioration of negative-ion production efficiency should occur after $t=100 \mathrm{~s}$ due to the ex- 


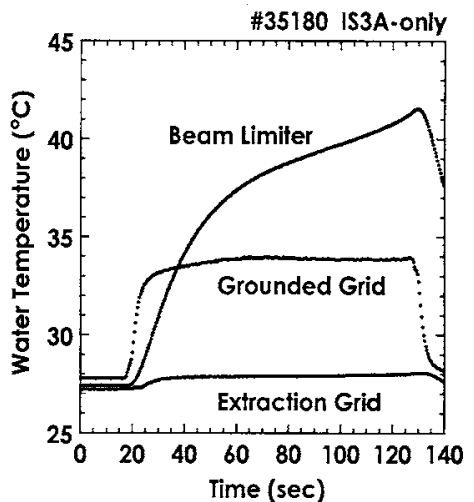

FIG. 4. Water temperature rises of the extraction grid, the grounded grid and the beam limiter at the entrance of the neutralizer in a long-pulse shot for $110 \mathrm{~s}$.

cessive temperature rise of the PG. Thus, the increase in the extraction current is thought to be derived from an increase in the electron current. Simultaneously the negative-ion current would be gradually reduced, resulting in enlargement of the beam divergence. Figure 4 shows the water temperature rises of the extraction grid, the grounded grid, and the beam limiter at the entrance of the neutralizer. After around $t=110 \mathrm{~s}$ the water temperature of the EG is raised, which should be ascribed to the increase in the electron current. The water temperature rise rate of the beam limiter is also increased after $t=110 \mathrm{~s}$, indicating the enlargement of the beam divergence. This degradation of the beam optics causes breakdowns between the grids, and the beam injection terminates at $t=125 \mathrm{~s}$ in Fig. 3, which corresponds to $110 \mathrm{~s}$ of the injection duration.

To extend the injection duration further and simultaneously to increase the injection power in long-pulse shots, the cooled PG, described in Sec. II, is used. As a result, the injection duration was extended to $128 \mathrm{~s}$ with an injection energy and power of $90 \mathrm{keV}$ and about $200 \mathrm{~kW}$, respectively, using one ion source. In this case, the injection was manually stopped due to the radiation collapse of the target plasma. Since the PG temperature rise was not saturated, the injection duration would be limited to about $3 \mathrm{~min}$ with the cooled PG even if the plasma did not collapse. Figure 5

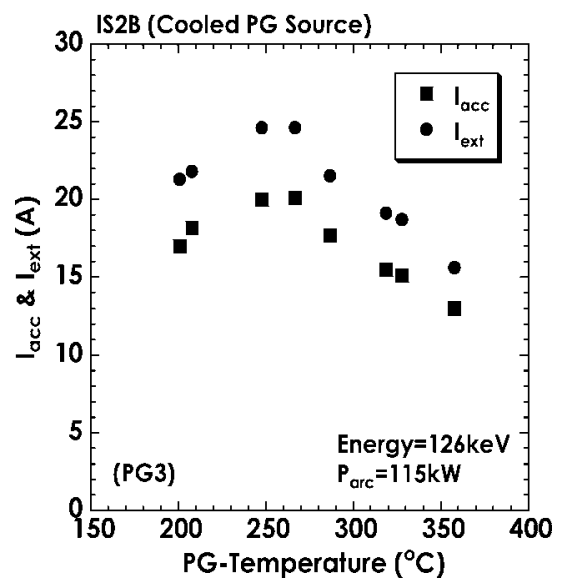

FIG. 5. Acceleration current and extraction current as functions of the plasma grid temperature in a source with the cooled plasma grid.

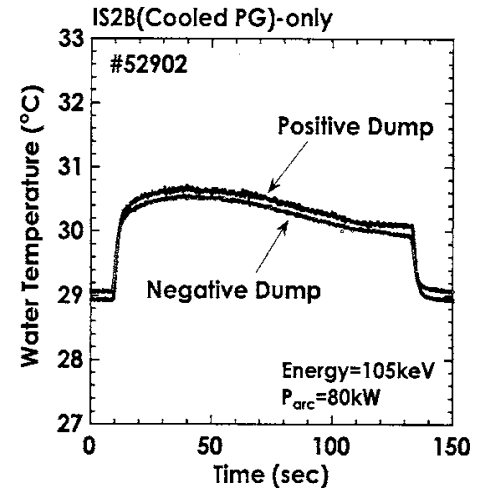

FIG. 6. Time variations of the output temperature of cooling water for the positive and negative beam dumps in a long-pulse shot for $124 \mathrm{~s}$. The injection power is about $250 \mathrm{~kW}$ and the arc power is $80 \mathrm{~kW}$.

shows the acceleration and extraction currents as functions of the PG temperature in a source with the cooled PG. The data are taken from a series of short-pulse shots at the same arc power, and the PG temperature is varied with the prearc duration and the shot interval. The dependency of these currents on the PG temperature should reflect that of the negative-ion production efficiency. At around $250{ }^{\circ} \mathrm{C}$ of the PG temperature the efficiency shows a maximum, and the appropriate operational range is thought to be $200-300{ }^{\circ} \mathrm{C}$. This tendency is similar to that of the source with the uncooled PG for the short-pulse operation. Time variations of the output temperature of cooling water for the positive and negative beam dumps are shown in Fig. 6, during a longpulse shot for $124 \mathrm{~s}$ with an injection power of about 250 $\mathrm{kW}$. The arc power is $80 \mathrm{~kW}$. The beam dumps are installed for the residual positive and negative-ion beams after the neutralization of the negative-ion beams from the ion source, and actively water cooled. Thus, the time variation of the water temperature rise corresponds to that of the heat load by the residual ion beams in a time scale of a few seconds. The residual ion beams are gradually decreased after around $t=80 \mathrm{~s}$, meaning a gradual decrease in the negative-ion current produced in the ion source. Considering the PG temperature dependency on the arc duration shown in Fig. 2, the PG temperature is thought to exceed $250{ }^{\circ} \mathrm{C}$ at around $80 \mathrm{~s}$ of the pulse duration when the arc power is $80 \mathrm{~kW}$. The decrease in the negative-ion current is ascribed to the increase in the PG temperature from the appropriate temperature for the negative-ion production, and the time variation of the negative-ion current shown in Fig. 6 almost coincides with the dependency shown in Fig. 5. It is found that the PG temperature control is significantly important for the efficient negative-ion production in the cesium-seeded operation, independent of the beam-pulse duration. The cooled PG structure used here is not enough for the long-pulse operation because the thermal insulation between the cooling channel and the PG is considered for the short-pulse operation. The active heating and cooling structure to control the PG temperature is required for the actual long-pulse negative-NB injector.

On the other hand, the beam profile in the vertical direction is observed to be more uniform with the cooled PG than that with the uncooled PG. Figure 7 shows the vertical beam 


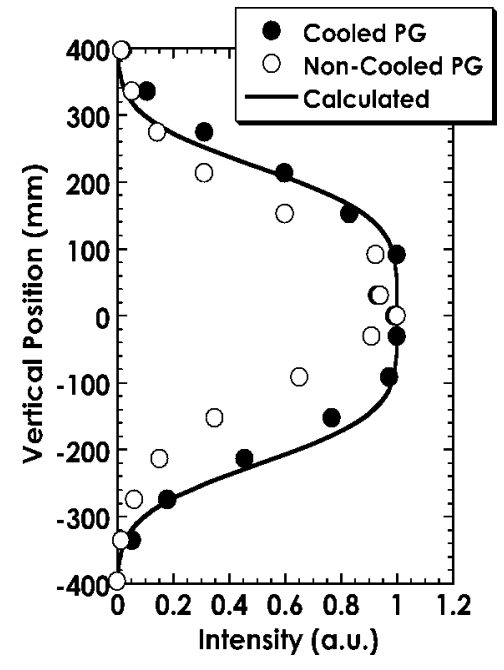

FIG. 7. Vertical beam profiles for sources with the cooled and uncooled PG, measured on the calorimeter $8.5 \mathrm{~m}$ downstream from the ion source. The calculated profile is also shown for a uniform beam with a divergence angle of $10 \mathrm{mrad}$ by a solid line in the figure.

profiles for the sources with the cooled and uncooled PG, measured by a calorimeter $8.5 \mathrm{~m}$ downstream from the ion source. The uniform beam profile can be obtained by adjusting the arc discharge distribution with the individual voltage control of the arc and filament power supplies, which are divided into 12 independently controlled circuits. ${ }^{6}$ However, the arc discharge distribution is adjusted here only with the individual variable resistances of the 12 divided outputs of the arc power supply. As shown in Fig. 7, the beam uniformity is much improved with the cooled PG, and it shows a similar vertical uniformity to that observed with the individual voltage control of the arc and filament power supplies. The temperature of the individual PG parts becomes more uniform with the cooled PG, which should contribute to the improvement of the beam uniformity.

\section{ACKNOWLEDGMENTS}

The authors acknowledge the LHD experimental group including all the technical staff for their excellent LHD-NBI experiments. The authors are grateful to Professor O. Motojima, Director General, for his continuous encouragement and support.

${ }^{1}$ Y. Takeiri et al., Proceedings of the Fourth IAEA Technical Meeting on Negative Ion Based Neutral Beam Injectors, Padova, Italy, 2005.

${ }^{2}$ O. Motojima et al., Phys. Plasmas 6, 1843 (1999).

${ }^{3}$ Y. Takeiri, M. Osakabe, K. Tsumori, Y. Oka, O. Kaneko, E. Asano, T. Kawamoto, R. Akiyama, and M. Tanaka, J. Plasma Fusion Res. 74, 1434 (1998).

${ }^{4}$ K. Tsumori et al., Fusion Energy 2004, Proceedings of the 20th International Conference, Vilamoura, 2004.

${ }^{5}$ Y. Takeiri et al., Plasma Phys. Controlled Fusion 42, 147 (2000).

${ }^{6}$ K. Ikeda et al., Rev. Sci. Instrum. 75, 1744 (2004). 